\title{
Formation of $p$-Unsubstituted Phenols in Base-catalyzed Lignin Depolymerization
}

\author{
Shinji KUDO ${ }^{1,2^{*}}$, Eriko HONDA ${ }^{1}$, Shingo NISHIOKA ${ }^{1}$, and Jun-ichiro HAYASHI ${ }^{1,2}$ \\ ${ }^{1}$ Interdisciplinary Graduate School of Engineering Sciences and ${ }^{2}$ Institute for Materials Chemistry and Engineering, Kyushu \\ University, 6-1 Kasuga Koen, Kasuga, 816-8580, Japan
}

\begin{abstract}
Lignin valorization is one of the biggest challenges for developing biomass-based chemical industry. Recent innovative works has enabled depolymerization of lignin to the monomers with the yield close to theoretical maximum. The main product is a mixture of phenols derived from the cleavage of $\beta-\mathrm{O}-4$ linkages between phenylpropanoid units. However, the obtained highly functionalized phenols are not common in current industry and, therefore, needs further processing for the utilization in intended applications. The present study shows that simple phenols without functional groups at para-position form selectively in base-catalyzed depolymerization of lignin. The yield is not sufficiently high, up to $6.9 \%$ on a carbon basis under the examined conditions, but the selectivity among released lignin monomers reaches $80 \%$. The study also discusses the mechanism of $p$-unsubstituted phenols formation.
\end{abstract}

\section{Introduction}

Lignocellulosic biomass is a renewable source of chemicals and fuels. The development of technologies for efficiently converting biomass to a variety of chemicals is a key to sustainable society with less dependency on fossil fuels. Studies on bio-renewable chemicals production so far mostly focussed on cellulose, a main component of biomass. On the other hand, to reach biochemical industry with economic feasibility, we need to consider valorization of the other portion of biomass.

Lignin accounts for 16-31 wt $\%$ of woody biomass. Although the detailed structure is different by the origin, lignin is a phenolic polymer with $\beta-\mathrm{O}-4$ as the most abundant linkage. Different from cellulose and hemicellulose, lignin is thus has a potential to be abundant direct source of bio-aromatics. However, the complex and recalcitrant structure inhibits the depolymerization to mono-aromatics. In fact, until several years ago, it was generally accepted that available yield of lignin monomers was limited to less than $10 \%$ (Beauchet et al., 2012). In recent years, triggered by key findings on the depolymerization chemistry (Song et al., 2013; Rahimi et al., 2014; Shuai et al., 2016), a rapid advancement has been made in this research field. There have been many studies that report near-complete lignin depolymerization. For example, Van den Bosch et al. (2017) showed that treatment of birch wood with $\mathrm{Ni} / \mathrm{Al}_{2} \mathrm{O}_{3}$ in methanol under pressurized hydrogen produced phenolic monomers with $44 \%$ yield, which was close to the theoretical maximum yield. Cellulose and hemicellulose in the residue were hardly damaged in the treatment and could be converted into bio-ethanol with $73 \%$ yield. This type of biorefinery strategy is called lignin-first fractionation, where depolymerization of native lignin from biomass and their quick stabilization are important for achieving high monomer yield. The depolymerization technology is thus being established at a high level. Whilst, the immediate utilization of obtained lignin oil is generally difficult because of their undesired properties consisting of a variety of highly functionalized phenols (Rinaldi et al., 2016). There are studies on downstream processing of the oil, but it often results in the utilization as a low-value added liquid fuel.

Hydrothermal base-catalyzed depolymerization is another approach to the lignin valorization (Vigneault et al., 2007; Beauchet et al., 2012; Katahira et al., 2016). Because it is a type of reaction occurring in traditional kraft process, chemistry behind the reaction has attracted attention for researchers. Brønsted basicity derived from base in water causes alteration of electronic structure of lignin and facilitates the dissolution and depolymerization (Otromke et al., 2019). Different from lignin-first strategy, depolymerized lignin is generally unstable, leading to the condensation and relatively low yields of soluble products (sulfide ion suppresses the condensation in kraft process). Even with the low yields, this approach is still attractive because of similarity to existing processes in paper industry. High dissolution power of basic water enables facile lignin extraction and downstream processing. In our previous studies, a process scheme for valorization of lignin utilizing base-catalyzed reaction was proposed, which consisted of three steps: lignin dissolved in basic water was depolymerized, lignin monomers were extracted from the aqueous phase with water-insoluble solvent, and the lignin left in aqueous phase was

\footnotetext{
Corresponding author: shinji_kudo@cm.kyushu-u.ac.jp
} 
completely reformed to combustible gas consisted mainly of methane (Kudo et al., 2014; Nishioka et al., 2014). Valorization of $78 \%$ lignin as monomers and fuel gas was demonstrated, and the basic water at the outlet could be fully recycled to the process.

A main focus of the present study is on the characteristics of lignin monomers from the basecatalyzed depolymerization. The monomers from ligninfirst fractionation consists mainly of phenols with propane, propylene, or propanol chain at para-position because of selective cleavage of $\beta-\mathrm{O}-4$ linkage. On the other hand, the monomers from base-catalyzed depolymerization tend to contain more $p$-unsubstituted phenols such as phenol, guaiacol, and syringol $(\mathrm{R}=\mathrm{H}$ in Figure 1). Those phenols have established market or high potential to be readily used in industry because of the simple structure. However, the formation of $p$-unsubstituted phenols is unusual because it generally involves C-C bond ( $\alpha-1$ linkage) cleavage, which needs much higher bond-dissociation energies than that of $\beta$-O-4 linkage (Galkin and Samec, 2016). Studies on chemistry of kraft pulping (Gierer, 1980) have evidenced the cleavage of $\alpha-1$ linkage but described that the $\mathrm{C}-\mathrm{C}$ bond cleavage accounts for only small portion of lignin depolymerization. In this study, the selective formation of $p$-unsubstituted phenols is demonstrated using two types of technical lignin in the base-catalyzed depolymerization, and discussion is made for providing insight into the mechanism of their formation.

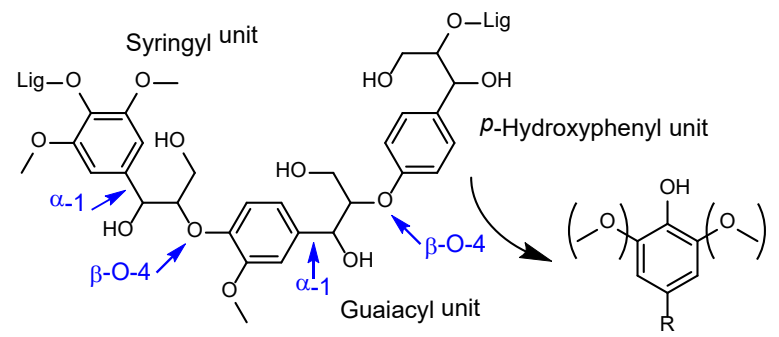

Figure 1. Simplified structure of lignin and lignin monomer

\section{Experimental}

\subsection{Materials}

Two types of lignin were used as feedstock. Lig-A was purchased form Sigma-Aldrich (lignin, alkali), and Lig-B was kindly supplied by Sanuki Kasei Co., Ltd., Japan. The ratio of aromatic nuclei, analysed by a common nitrobenzene oxidation method (Rodrigues et al., 2011), was syringyl $/$ guaiacyl $/ p$-hydroxyphenyl $(\mathrm{mol} \%)=1 / 94 / 5$ and 25/48/26 for Lig-A and Lig-B, respectively.

\subsection{Reaction and product analysis}

Experiments of base-catalyzed lignin depolymerization were carried out mainly with a flow type reactor (Figure 2). $\mathrm{Na}_{2} \mathrm{CO}_{3}$ was used to make basic solution. $\mathrm{NaOH}$ is often used for the base-catalyzed depolymerization, but it reacts with generated carbonate ion during the reaction to form $\mathrm{Na}_{2} \mathrm{CO}_{3}$. For the direct reuse to the process, $\mathrm{Na}_{2} \mathrm{CO}_{3}$ would be a better choice (Kudo et al., 2014). $0.1 \mathrm{M}$ $\mathrm{Na}_{2} \mathrm{CO}_{3}$ aqueous solution was fed to the tubular reactor (SUS316, i.d. $2.17 \mathrm{~mm}$, volume $9 \mathrm{~mL}$ ) at $11 \mathrm{MPa}$, and the reactor was heated to a prescribed temperature. Then, the feed was switched to a solution containing lignin at 5,000 ppm (mg-C/L) in $0.1 \mathrm{M} \mathrm{Na}_{2} \mathrm{CO}_{3}$ aqueous solution. The reaction time was controlled by feeding rate of the lignin solution. The depolymerization experiment was also carried out with a batch reactor. A container $(10 \mathrm{~mL})$ consisting of Swagelok fittings was loaded with lignin, 5 $\mathrm{mL}$ of $0.1 \mathrm{M} \mathrm{Na}_{2} \mathrm{CO}_{3}$ aqueous solution or water, and nitrogen (ambient pressure), and submerged in a fluidized sand bath heated at a reaction temperature. After a prescribed reaction time, the reaction was quenched by immersing the reactor in an iced water.

The product solution was acidified to $\mathrm{pH}=1-1.5$ with $\mathrm{HCl}$, and separated by PTFE membrane filter (pore size: $0.45 \mu \mathrm{m}$ ) into acid-soluble and -insoluble fractions. The acid-soluble fraction was extracted by ethyl acetate and subjected to gas chromatography-mass spectrometry (GC-MS) analysis with a PerkinElmer Clarus SQ8 for the quantification of lignin monomers. Details for the analytical method can be found elsewhere (Kudo et al., 2014). The acid-insoluble fraction (acid-insoluble lignin: AIL) was dried in vacuo at $40^{\circ} \mathrm{C}$ overnight. AIL was analysed by thermogravimetry (TGA), Fourier transform infrared spectroscopy (FT-IR), and gel permeation chromatography (GPC). TGA was performed on a SII Nano Tecnhology EXSTAR TG/DTA 7200. 2-2.5 mg of AIL was heated at $5^{\circ} \mathrm{C} / \mathrm{min}$ under nitrogen flow (200 $\mathrm{mL} / \mathrm{min})$. The conversion $(X)$ was calculated with a relative mass on a basis of the mass at $110^{\circ} \mathrm{C}$. FT-IR spectra were measured on a PerkinElmer, Spectrum two equipped with an attenuated total reflectance (ATR) cell at $4 \mathrm{~cm}^{-1}$ resolution. GPC analysis was carried out with a Shimadzu LG-20 prominence series equipped with three columns connected in series (Tosoh TSKgel G3000HXL, G2000HXL, and G1000HXL) using tetrahydrofuran (THF) as mobile phase. Before GPC analysis, AIL samples were acetylated with acetic anhydride in pyridine to be soluble in THF.

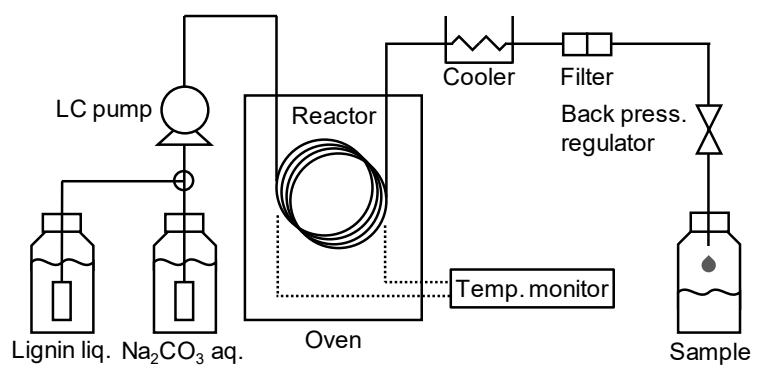

Figure 2. Schematic diagram of experimental setup for basecatalyzed lignin depolymerization in a flow type reactor

\section{Results and Discussion}

\subsection{Effect of base-catalysis on depolymerization}

To investigate the effect of base-catalysis, hydrothermal lignin depolymerization was carried out in $0.1 \mathrm{M} \mathrm{Na}_{2} \mathrm{CO}_{3}$ 
and water. The batch reactor was employed for this experiment because lignin was little soluble in water to feed into the flow reactor. The difference of depolymerized products was apparent from GC-MS chromatograms (Figure 3). Both products contained phenol, guaiacol, and syringol, but the depolymerization in water also produced phenolic compounds ( $\mathrm{Phs)}$ with substituent at para-position. Figure 4 shows the yields of Phs from Lig-A and Lig-B. The main Phs from basecatalyzed depolymerization were guaiacol for Lig-A and phenol, guaiacoal, and syringol for Lig-B. The compositions agree with their aromatic nuclei structure. Lig-A is from softwood and consists mainly of guaiacyl unit. Lig-B is from bamboo and has all three units (syringyl, guaiacyl, $p$-hydroxyphenyl). $p$-Substituted Phs (= other Phs) accounted for only a minor portion of the lignin monomers. Moreover, the total yield of Phs was much higher for the depolymerization under basecatalysis, reaching $6.9-8.4 \%$ on a carbon basis $(\%-C)$, although it is not necessarily high, compared to that in recent studies, which achieved near-complete lignin depolymerization (Song et al., 2013; Rahimi et al., 2014; Shuai et al., 2016). It was thus confirmed for two types of lignin that base-catalyzed depolymerization selectively produces $p$-unsubtituted Phs.
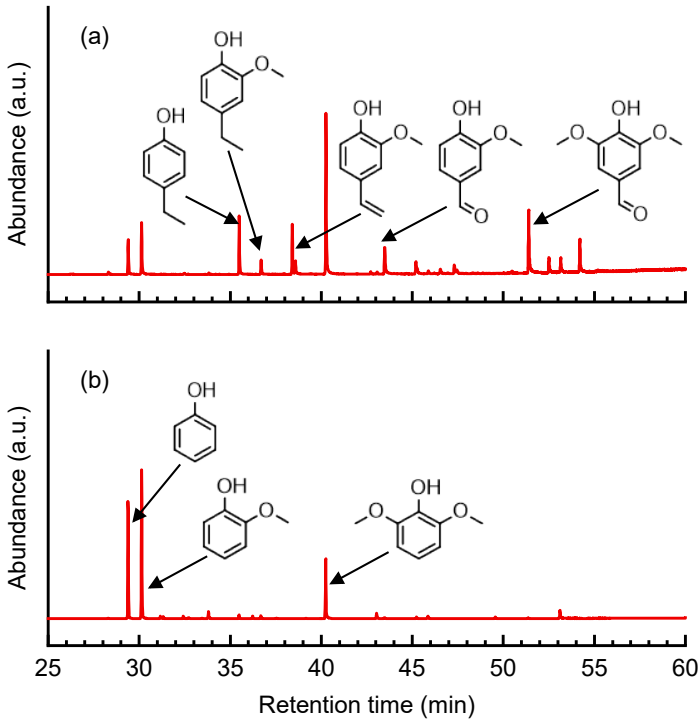

Figure 3. GC-MS chromatograms of acid-soluble fraction of Lig-B after depolymerization in (a) water and (b) 0.1 $\mathrm{M} \mathrm{Na}_{2} \mathrm{CO}_{3}$ at $300^{\circ} \mathrm{C}$ for $20 \mathrm{~min}$ in batch reactor

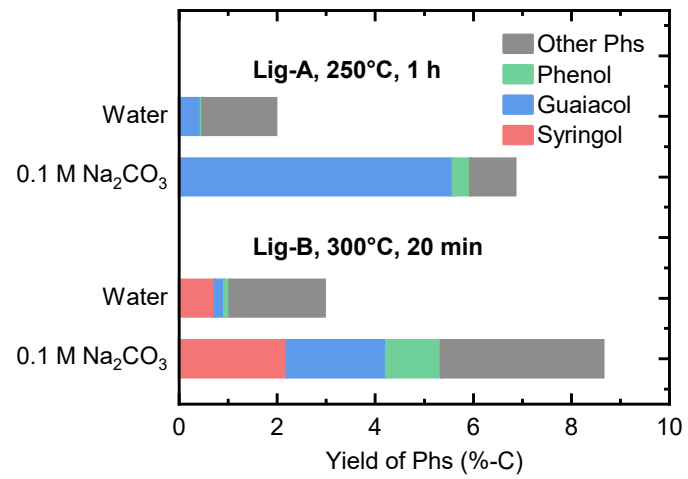

Figure 4. Yield of lignin monomers (Phs) from Lig-A and Lig$\mathrm{B}$ after depolymerization in batch reactor

\subsection{Base-catalyzed lignin depolymerizaiton in flow type reactor}

The base-catalyzed depolymerization was carried out in the flow type reactor for investigating the effect of reaction time on the products in detail. The total $\mathrm{Phs}$ yields are presented in Figure 5. The yield increased with reaction time and reached the maximum at 4-9 min in the reaction at $300^{\circ} \mathrm{C}$. As with the batch experiment, $\mathrm{Phs}$ consisted mainly of phenol, guaiacol, and syringol. Their yields were 5.5 and $6.9 \%$-C, and selectivities among Phs were 61.0 and $80.0 \%$ for Lig-A and Lig-B, respectively, at the maximum. The proportion of aromatic carbon, analysed by solid state ${ }^{13} \mathrm{C} \mathrm{NMR}$, in the total carbon of Lig-B was 54.1\%. Therefore, the yield showed substantial portion of aromatics in Lig-B, more than $10 \%$, was released as $p$-unsubtituted Phs.

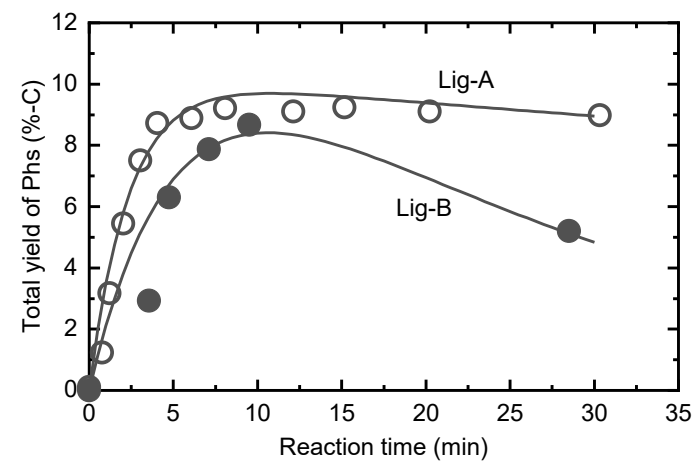

Figure 5. Total yield of $\mathrm{Phs}$ at different reaction time: lignin 5,000 ppm, $0.1 \mathrm{M} \mathrm{Na}_{2} \mathrm{CO}_{3}, 300^{\circ} \mathrm{C}, 11 \mathrm{MPa}$, flow reactor

After showing the maximum, the total yield decreased for Lig-B in particular. To investigate the reason for the decreasing trend, the stability of main phenols under basecatalyzed condition was investigated (Figure 6). In the reaction at $325^{\circ} \mathrm{C}$, phenol was relatively stable, losing only $13 \%$ of its portion even at $11 \mathrm{~min}$. On the other hand, major portion of guaiacol and syringol were converted to degradation products. A linear increase in the conversion presented independency of kinetics on the concentration and indicated the influence of base-catalysis. Under the basic condition, guaiacol undergoes hydrolysis of methoxyl group to form catechol (Beauchet et al., 2012). Similarly, syringol is demethoxylated to form guaiacol and then catechol. However, this does not explain the decrease in the total yield of Phs at a long reaction time. A plausible reason is condensation of the phenols, but the formation of condensed solid material was not visually observed in the experiment of Figure 6 .

Detected Phs from the reaction were 21 and 37 types for Lig-A and Lig-B, respectively. Figure 7 summarizes temporal change in the yield of Phs, which showed the highest yield over $0.2 \%$-C, for Lig-A. As seen from the result, ether linkage or hydroxyl groups at $\mathrm{C} \alpha$ and $\mathrm{C} \gamma$ position did not survive the reaction. Even including other Phs, no Phs contained hydroxyl group in $p$-substituents. This was because of catalysis induced by Brønsted basicity for the deprotonation. Because the catalysis was active toward a wide variety of linkages in lignin, many types of Phs were formed as primary products. Therefore, 
the most Phs were produced at an early stage of the reaction excepting catechol that was formed from guaiacol. In kraft process, sulfidolytic cleavage also helps the depolymerization, making the composition of Phs more complicated (Gierer, 1980).

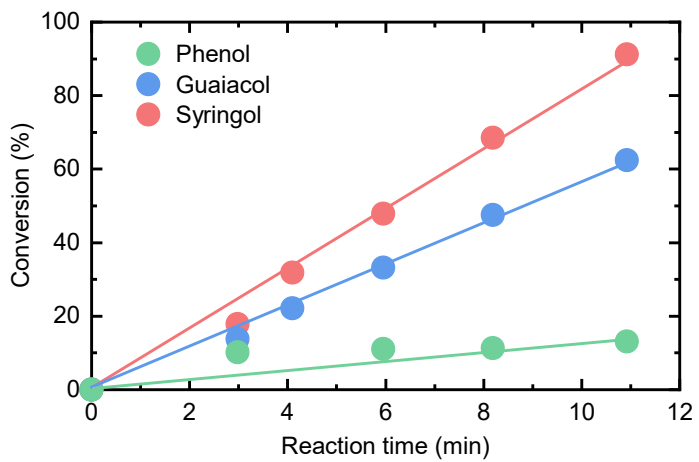

Figure 6. Stability of phenol, guaiacol, and syringol under base-catalyzed condition: phenols $500 \mathrm{ppm}, 0.1 \mathrm{M}$ $\mathrm{Na}_{2} \mathrm{CO}_{3}, 325^{\circ} \mathrm{C}, 11 \mathrm{MPa}$, flow reactor

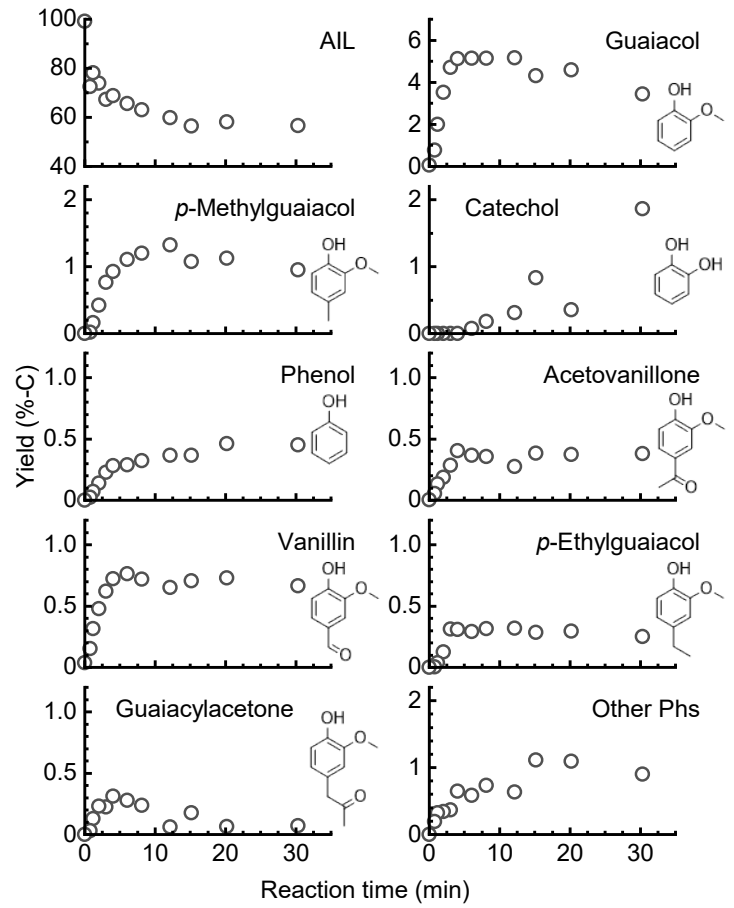

Figure 7. AIL yield and breakdown of Phs yield from Lig-A: lignin 5,000 ppm, $0.1 \mathrm{M} \mathrm{Na}_{2} \mathrm{CO}_{3}, 300^{\circ} \mathrm{C}, 11 \mathrm{MPa}$, flow reactor

\subsection{Analysis of AIL}

Figure 7 also shows the yield of AIL. Raw Lig-A (reaction time $=0 \mathrm{~min}$ ) consisted mostly of AIL with the recovery of $99.1 \%$-C. The AIL recovery dropped to $72.5 \%-\mathrm{C}$ at the initial stage of the reaction $(0.8 \mathrm{~min})$ and gradually decreased to $56.6 \%$-C at $30 \mathrm{~min}$. The sum of yields of AIL and $\mathrm{Phs}$ were in the range of $65.6-79.3 \%-\mathrm{C}$ at $0.8-30 \mathrm{~min}$, and thus indicated the presence of acid-soluble products that could not be detected in GC-MS analysis and had oligomeric structure. In other words, the oligomeric product, accounting for over $20 \%$-C portion of Lig-A, was released even with the short reaction time of $0.8 \mathrm{~min}$.
Because the yield of Phs more slowly increased, the fragmented oligomer was unlikely the source of $p$ unsubstituted Phs. This observation in turn suggested that the residual phase was gradually reformed to quinone methide intermediates, which was active to retrograde aldol reactions (Gierer, 1980), releasing $p$-unsubstituted Phs. Because the present reaction conditions were not sufficient for actively causing the cleavage of $\beta$-O-4 linkage, $p$-unsubstituted $\mathrm{Phs}$ accounted for major portion of $\mathrm{Phs}$ in the product.

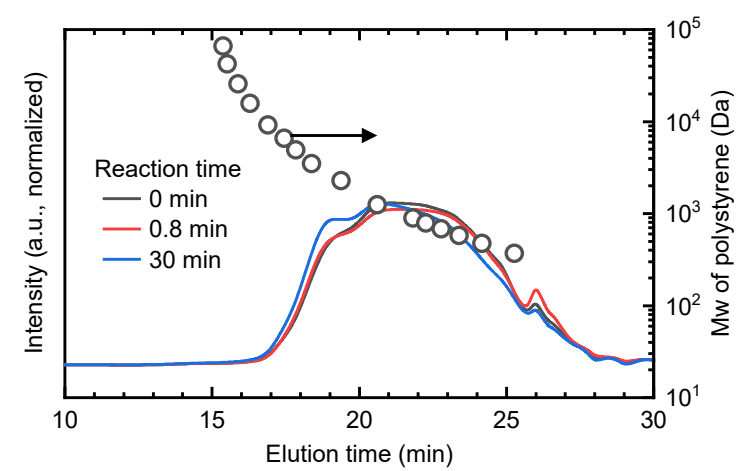

Figure 8. GPC chromatograms of AIL after base-catalyzed depolymerization for $0,0.8$, and $30 \mathrm{~min}$.

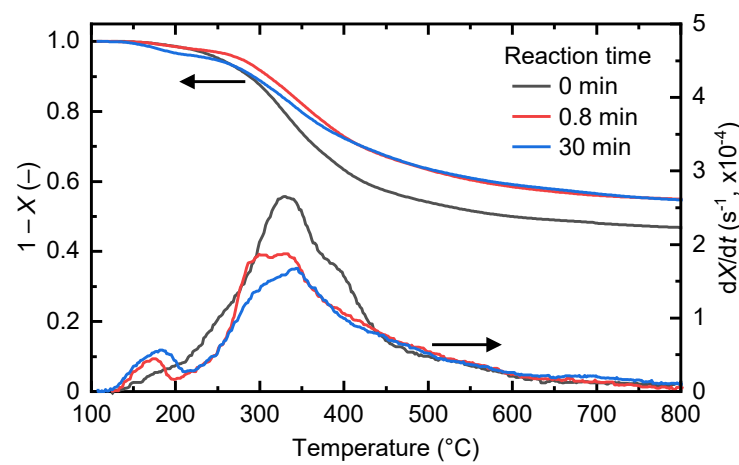

Figure 9. TG curves and $\mathrm{d} X / \mathrm{d} t$ profiles of AIL after basecatalyzed depolymerization for $0,0.8$, and $30 \mathrm{~min}$.

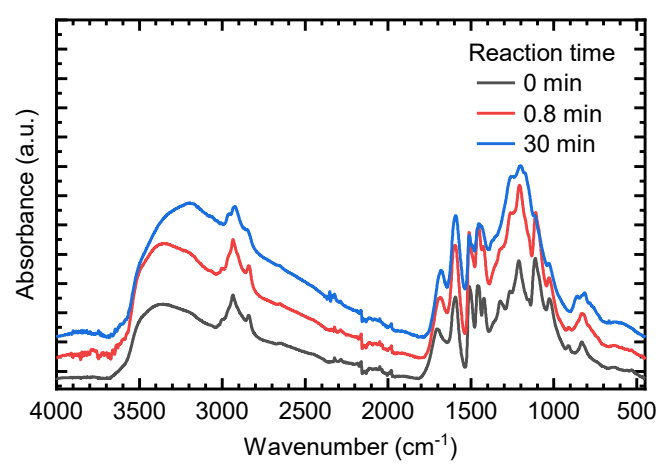

Figure 10. FT-IR spectra of AIL after base-catalyzed depolymerization for $0,0.8$, and $30 \mathrm{~min}$.

Selected AIL samples were analysed by GPC, TGA, and FT-IR. There was little difference in GPC chromatograms of AIL after $0,0.8$, and 30 min of reaction (Figure 8 ). The base-catalysis depolymerizes lignin while promotes the condensation in parallel, which resulted in apparent similarity in the molecular size distribution. The carbon content, measured by elemental analysis, was 63.3 , $70.2,71.6 \mathrm{wt} \%$ for AIL with the reaction time of $0,0.8$, 
and $30 \mathrm{~min}$, respectively, supporting the progress of condensation. TGA also showed the effect of reactions induced by the base-catalysis (Figure 9). TG curves presented the decrease in volatile matter content. The concave in $\mathrm{d} X / \mathrm{d} t$ profiles around $200-300^{\circ} \mathrm{C}$ was supposedly caused by the release of acid-soluble oligomeric fraction. On the other hand, FT-IR analysis (Figure 10) distinguished the structures of AIL with the reaction time of 0.8 and $30 \mathrm{~min}$. All spectra contained IR absorbance at wavenumbers characteristic of lignin, but the peak at $1060-1160 \mathrm{~cm}^{-1}$, corresponding to the ether linkage, was hardly observed only for AIL after $30 \mathrm{~min}$ of the reaction. This indicated that precursor of $p$ unsubstituted Phs still survived after the initial release of its large portion as acid-soluble fraction and gradually decreased along with the formation of Phs. According to the above mentioned mechanism of $\alpha$-1 linkage cleavage (Gierer, 1980), the phenolic unit of $p$-unsubstituted Phs precursor needs to be phenolate anion with $\beta$-O-4 linkage at $\mathrm{C} \beta$ position. Given the yield of $p$-unsubstituted $\mathrm{Phs}$, the precursor-like structure dominated at least more than $10 \%$ of aromatic rings in Lig-A.

\section{Conclusions}

Base-catalyzed depolymerizaiton experiments using two types of technical lignin demonstrated the effectiveness for selectively producing $p$-unsubstituted Phs such as phenol, guaiacol, and syringol. The yield reached 5.5$6.9 \%-\mathrm{C}$ in the short reaction time of $5-9 \mathrm{~min}$ at $300^{\circ} \mathrm{C}$, and the selectivity among lignin monomers was 61.0 $80.0 \%$. In the reaction, more than $20 \%$-C of lignin was released as acid-soluble lignin oligomer at the initial stage. The residual phase retained abundant precursor of $p$ unsubstituted Phs, and presumably formed phenolate anion linked to lignin molecule, followed by the cleavage of $\beta$-O-4 linkage. Under the base-catalysis, the released phenol was relatively stable, but guaiacol and syringol were converted to degradation products with a long reaction time.

\section{Acknowledgements}

This work was performed under the Cooperative Research Program of the "Network Joint Research Center for Materials and Devices".

\section{References}

Beauchet, R., F. Monteil-Rivera, and J. M. Lavoie; "Conversion of Lignin to Aromatic-based Chemicals (Lchems) and Biofuels (L-fuels)," Bioresour. Technol., 121, 328-334 (2012)

Galkin, M. V. and J. S. Samec; "Lignin Valorization Through Catalytic Lignocellulose Fractionation: A Fundamental Platform for the Future Biorefinery," ChemSusChem, 9, 1544-1558 (2016)

Gierer, J.; "Chemical Aspects of Kraft Pulping," Wood Sci. Technol., 14 (4), 241-266 (1980)
Katahira, R., A. Mittal, K. McKinney, X. Chen, M. P. Tucker, D. K. Johnson, and G. T. Beckham; "BaseCatalyzed Depolymerization of Biorefinery Lignins," ACS Sustainable Chem. Eng., 5, 1474-1486 (2016)

Kudo, S., Y. Hachiyama, Y. Takashima, J. Tahara, S. Idesh, K. Norinaga, and J.-i. Hayashi; "Catalytic Hydrothermal Reforming of Lignin in Aqueous Alkaline Medium,” Energy Fuels, 28, 76-85 (2014)

Nishioka, S., S. Kudo, Y. Takashima, Y. Hachiyama, K. Norinaga, and J.-i. Hayashi; "Hydrothermal Conversion of Lignin to Monomeric Phenols and Fuel Gas Using Alkaline Aqueous Solution," Proc. of the 4th ASCONIEEchE 2014, pp. 56-61, Hualien, Taiwan (2014)

Otromke, M., R. J. White, and J. Sauer; "Hydrothermal Base Catalyzed Depolymerization and Conversion of Technical Lignin - An Introductory Review," Carbon Resour. Conv., 2, 59-71 (2019)

Rahimi, A,. A. Ulbrich, J. J. Coon, S. and S. Stahl; "Formic-acid-induced Depolymerization of Oxidized Lignin to Aromatics," Nature, 515, 249-252 (2014)

Rinaldi, R., R. Jastrzebski, M. T. Clough, J. Ralph, M. Kennema, P. C. Bruijnincx, and B. M. Weckhuysen; "Paving the Way for Lignin Valorisation: Recent Advances in Bioengineering, Biorefining and Catalysis," Angew. Chem. Int. Ed., 55, 8164-8215 (2016)

Rodrigues Pinto, P. C., E. A. Borges da Silva, and A. E. Rodrigues; "Insights into Oxidative Conversion of Lignin to High-Added-Value Phenolic Aldehydes," Ind. Eng. Chem. Res., 50, 741-748 (2011)

Shuai, L., M. T. Amiri, Y. M. Questell-Santiago, L. Héroguel, Y. Li, H. Kim, R. Meilan, C. Chapple, J. Ralph, and J. S. Luterbacher; "Formaldehyde Stabilization Facilitates Lignin Monomer Production During Biomass Depolymerization," Science, 354, 329-333 (2016)

Song, Q., F. Wang, J. Cai, Y. Wang, J. Zhang, W. Yu, and J. Xu; "Lignin Depolymerization (LDP) in Alcohol Over Nickel-based Catalysts via a FragmentationHydrogenolysis Process," Energy Environ. Sci., 6, 9941007 (2013)

Van den Bosch, S., T. Renders, S. Kennis, S. F. Koelewijn, G. Van den Bossche, T. Vangeel, A. Deneyer, D. Depuydt, C. M. Courtin, J. M. Thevelein, W. Schutyser, and B. Sels; "Integrating Lignin Valorization and Bio-ethanol Production: on the Role of $\mathrm{Ni}-\mathrm{Al}_{2} \mathrm{O}_{3}$ Catalyst Pellets during Lignin-first Fractionation," Green Chem., 19, 3313-3326 (2017)

Vigneault, A., D. K. Johnson, and E. Chornet; "BaseCatalyzed Depolymerization of Lignin: Separation of Monomers," Can. J. Chem. Eng., 85, 906-916 (2007) 